\title{
Onset of Giant Planet Migration before 4480 Million Years Ago
}

\author{
Stephen J. Mojzsis ${ }^{1,2}$ (10, Ramon Brasser ${ }^{3}$, Nigel M. Kelly ${ }^{1,6}$, Oleg Abramov ${ }^{4}$, and Stephanie C. Werner ${ }^{5}$ \\ ${ }^{1}$ Department of Geological Sciences, University of Colorado, UCB 399, 2200 Colorado Avenue, Boulder, CO 80309-0399, USA; mojzsis@colorado.edu \\ ${ }^{2}$ Institute for Geological and Geochemical Research, Research Centre for Astronomy and Earth Sciences, Hungarian Academy of Sciences, 45 Budaörsi Street, \\ H-1112 Budapest, Hungary \\ ${ }^{3}$ Earth Life Science Institute, Tokyo Institute of Technology, Ookayama, Meguro-ku, Tokyo 152-8550, Japan \\ ${ }_{4}^{4}$ Planetary Science Institute, 1700 East Fort Lowell Road, Suite 106, Tucson, AZ 85719, USA \\ ${ }^{5}$ Centre for Earth Evolution and Dynamics, University of Oslo, Postbox 1028 Blindern, 0316 Oslo, Norway \\ Received 2019 March 20; revised 2019 June 18; accepted 2019 June 19; published 2019 August 12
}

\begin{abstract}
Soon after their formation, the terrestrial planets experienced intense impact bombardment by comets, leftover planetesimals from primary accretion, and asteroids. This temporal interval in solar system evolution, termed late accretion, thermally and chemically modified solid planetary surfaces and may have impeded life's emergence on the Hadean (pre-3850 Ma) Earth. The sources and tempo of bombardment, however, remain obscure. Here we present a timeline that relates variably retentive radiometric ages documented from asteroidal meteorites to new dynamical models that invoke an early episode of planetesimal-driven giant planet migration after the dispersal of the protoplanetary disk. Reconciliation of geochronological data with dynamical models shows that such giant planet migration should lead to an intense $\sim 30 \mathrm{Myr}$ influx of comets to the entire solar system manifested in radiometric age data. The absence of wholesale crustal reset ages after $\sim 4450$ Ma for the most resilient chronometers from Earth, Moon, Mars, 4 Vesta, and various meteorite parent bodies confines the onset of giant planet migration to ca. $4480 \mathrm{Ma}$. Waning impacts continue to strike the inner planets through a protracted monotonic decline in impactor flux, in agreement with predictions from crater chronology. New global 3D thermal analytical bombardment models derived from our revised impact mass-production functions show also that persistent niches for prebiotic chemistry leading to the emergence of life on the early Hadean Earth could endure late accretion since at least about 4400 million years ago.
\end{abstract}

Key words: planets and satellites: dynamical evolution and stability - meteorites, meteors, meteoroids - minor planets, asteroids: general

Supporting material: data behind figure, tar.gz

\section{Introduction}

The nature of early bombardment to the inner solar system is a long-standing problem in planetary science. We use the term late accretion to refer to protracted and ongoing bombardment wherein leftover material composed of comets, planetesimals, and asteroids continues to strike the planets after their formation (Table 1). Although a matter of debate, a signal of this bombardment is traced by the chondritic relative abundances of the highly siderophile elements (HSEs, which include the platinum group elements: $\mathrm{Os}, \mathrm{Ir}, \mathrm{Ru}, \mathrm{Rh}, \mathrm{Pt}$, and Pd), regarded as pollutants by the earliest phase of late accretion to a planet's mantle (Walker 2009); these elemental abundances are in turn used to infer an exogenous mass augmentation to the terrestrial planets after separation of their cores and mantles (e.g., Bottke et al. 2010).

There exist two competing paradigms to describe the timedependent flux of late accretion. The first argues for a burst of relatively short duration in the intensity of impact bombardment hailed as a "lunar cataclysm" or, more commonly, "late heavy bombardment" (LHB); this is assumed to have affected the whole inner solar system (Morbidelli et al. 2012, 2018 and references therein; see Ryder 2002) at around $3950 \mathrm{Ma}$, or perhaps earlier. The LHB is largely implied by interpretations of uncorrected ${ }^{40-39} \mathrm{Ar}$ chronology from lunar samples (e.g., Fernandes et al. 2013) and has been promulgated by several

\footnotetext{
${ }^{6}$ Present address: Bruker Nano Analytics, Am Studio 2D, D-12489 Berlin, Germany.
}

popular dynamical models involving late-stage giant planet migration (e.g., Gomes et al. 2005). Alternatively, the second concept proposes an overall monotonic decline in the bombardment rate, which is an interpretation most broadly consistent with crater counting studies (Hartmann 1970; Neukum et al. 1975; see Werner \& Ivanov 2015 for a recent review).

To transcend the inconsistency between the two different models for late accretion cited above-which also has a strong bearing on the Hadean Earth's proclivity to host a nascent biosphere (e.g., Maher \& Stevenson 1988; Fritz et al. 2014)requires a comprehensive review of geochronological data for the inner solar system and a new investigation of planetary dynamics for the outer solar system.

\section{Late Accretion: Monotonic Decline or Late Heavy Bombardment?}

Direct records of the earliest phases of solar system evolution that could shed light on the inception of late accretion in the first $\sim 100 \mathrm{Myr}$ are either rare or absent from the crusts of the Moon, Earth, and Mars. On Earth, this deficit has been ascribed to endogenous crust-mantle interactions that recycled the primary crust (e.g., Hopkins et al. 2008, 2010), or less popularly, to the destructive thermal and mechanical effects of impact bombardments that accompanied the earliest stages of late accretion. If intense enough (such as in the event at ca. $4500 \mathrm{Ma}$ attributed to the "late veneer"; Genda et al. 2017), late accretion can result in melting and isotopic 
Table 1

Terms, Definitions, and Relevant Geological and Cosmochemical Time Intervals Used in This Work

\begin{tabular}{|c|c|c|}
\hline Terms Used Here & Definition & Relevant Time Period in Solar System Evolution \\
\hline Primary accretion & $\begin{array}{l}\text { Accumulation of particles by gravitational attraction in an } \\
\text { accretion disk that ultimately led to planet formation }\end{array}$ & First several tens of millions of years \\
\hline Grand Tack & Early migration of Jupiter & First few million years \\
\hline Giant Impact (GI) & Formation of Earth's Moon by a large ( $\sim$ Mars mass?) body & $4510 \mathrm{Ma}$ (perhaps later) \\
\hline Late Veneer (LV) & $\begin{array}{l}\text { Mass augmentation of Earth, Moon and Mars by chondritic } \\
\text { materials after core closure; traced by highly siderophile } \\
\text { elements }\end{array}$ & After GI; approximately $4500-4480 \mathrm{Ma}$ \\
\hline $\begin{array}{l}\text { Late heavy bombardment (LHB), } \\
\text { or lunar cataclysm }\end{array}$ & $\begin{array}{l}\text { Purported "spike" in impacts at } \sim 3950 \mathrm{Ma} \text {, or earlier } \\
\quad(4100-4400 \mathrm{Ma}), \text { attributed to late giant planet migration }\end{array}$ & $\begin{array}{l}\text { Generally unconstrained but tied to Nice model; } \\
3800-4500 \mathrm{Ma}\end{array}$ \\
\hline Late accretion & $\begin{array}{l}\text { All post-GI bombardment up to the present day, including } \\
\text { the consequences of giant planet migration }\end{array}$ & $\sim 4480$-now \\
\hline $\begin{array}{l}\text { Nice model (giant planet } \\
\text { migration) }\end{array}$ & $\begin{array}{l}\text { Migration of the giant planets from an initial compact con- } \\
\text { figuration into their present positions }\end{array}$ & $\begin{array}{l}\text { Nice model was motivated by the LHB at ca. } 3.95 \mathrm{Ga} \text {. In this } \\
\text { work, its preferred timing linked with giant planet migration } \\
\text { is before } 4480 \mathrm{Ma} \text {. }\end{array}$ \\
\hline Hadean eon & $\begin{array}{l}\text { The first eon of Earth history that witnessed planet formation, } \\
\text { the origin of the crust, atmosphere, and oceans, as well as } \\
\text { life's emergence }\end{array}$ & $4567-3850 \mathrm{Ma}$ \\
\hline
\end{tabular}

rehomogenization of the crust and mantle that compose the planet's silicate reservoirs (Allègre et al. 2008). As a matter of fact, several lines of evidence exist that Earth's terrestrial silicate reservoirs experienced such a re-equilibration event well after cessation of primary accretion (Roth et al. 2014). This is attested by analysis of terrestrial samples using the timesensitive radiogenic tracers of lithosphere production and destruction such as ${ }^{182} \mathrm{Hf}-{ }^{182} \mathrm{~W},{ }^{235,238} \mathrm{U}-{ }^{207,200} \mathrm{~Pb},{ }^{129} \mathrm{I}-{ }^{129} \mathrm{Xe}$, and ${ }^{146} \mathrm{Sm}-{ }^{142} \mathrm{Nd}$ (e.g., Albarède \& Juteau 1984; Allègre et al. 1995, 2008; Zhang 2002). For the Moon, the mere existence of ancient lunar zircons (zirconium orthosilicate, or $\mathrm{Zr}\left(\mathrm{SiO}_{4}\right)$ ) proves that its crust was not completely melted by endogenous processes such as impacts, at least since ca. $4417 \mathrm{Ma}$ (Nemchin et al. 2009) and likely earlier. Thus far, direct records (as opposed to model ages) of pre-4.42 Ga lunar materials appear to have been destroyed even as evidence accumulates that the Moon experienced ruinous basin-forming events before 4100 Ma (Norman \& Nemchin 2014). Indirect evidence from coupled $\mathrm{U}-\mathrm{Pb}$ and Hf-isotopes also shows that significant differentiation and crust formation on the Moon commenced at ca. $4510 \mathrm{Ma}$, commensurate with the proposed time of the Moon's own formation (Barboni et al. 2017). Remote sensing studies reveal that Venus's crust may be no older than about $1000 \mathrm{Ma}$ (McKinnon et al. 1997; see Rolf et al. 2018), and the oldest features on Mercury may be comparable in age to those of the Moon and Mars (Strom \& Neukum 1988; Marchi et al. 2013). The firmest minimum date for the perpetuation of the crusts of Earth and Mars comes from preserved ages for the oldest terrestrial zircons at ca. $4400 \mathrm{Ma}$ (Valley et al. 2014) and the oldest comparable Martian zircons, which have been dated at 4428-4470 Ma (Humayun et al. 2013; Bouvier et al. 2018). That these minerals survive at all demonstrates that the crusts of the sampled terrestrial worlds did not experience wholesale melting since that time (Figure 2 of Hopkins et al. 2015; Heisinger \& Head 2006). Thus, available geological and cosmochemical data from the terrestrial planets, which could directly bear on the temporal flux profile of late accretion, are confined to the last ca. $4450 \mathrm{Myr}$.

Earth's Moon shows an unusual history: impact-modified lunar samples returned by the Apollo and Luna missions from the lunar near side yield ${ }^{40-39} \mathrm{Ar}, \mathrm{U}-\mathrm{Pb}$, and $\mathrm{Rb}-\mathrm{Sr}$ ages that seem to cluster around $3950 \mathrm{Ma}$ (Turner et al. 1973; Tera et al. 1974); this

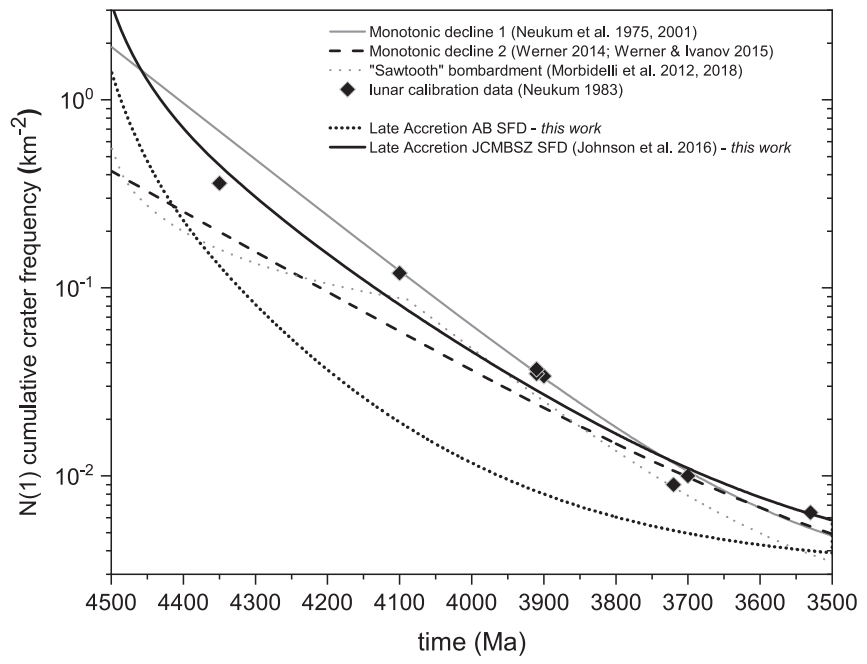

Figure 1. Lunar impact fluxes from various sources. The differential number of lunar craters with diameter $>1 \mathrm{~km} N(1)$ as a function of time and per unit surface for several scenarios discussed in the text. AB SFD = "asteroid belt size-frequency distribution." JCMBSZ SFD = "Johnson-Collins-MintonBowling-Simonson-Zuber size-frequency distribution" from Johnson et al. (2016). Data to reproduce these plots are provided in Supporting Information (Table S1).

(The data used to create this figure are available).

is far younger than the oldest documented ages of the lunar crust cited above (Nemchin et al. 2009; Barboni et al. 2017). Such a grouping of ages has long been used to argue that an LHB-type event affected the inner solar system as a rapid rise, and ensuing demise, in the impactor flux (Ryder 1990, 2002). Intriguingly, neither lunar, nor Mercurian, nor Martian crater chronologies show evidence for such an LHB event (Werner 2014), and it has been suggested that the 3950 Ma ages may instead be from a biased sampling compromised from widespread contamination of debris associated with the Moon's Imbrium basin (e.g., Shearer \& Borg 2006). Statistical analyses of crater distributions point instead to a monotonic decline in impactor flux from at least 4400 Ma (Figure 1). It is therefore equivocal whether there was an LHB at all, or that if there was, it occurred with as yet no clear temporal constraints (e.g., Hartmann 2003). 
Beyond Earth, the Moon, and Mars, highly retentive U-Pb and $\mathrm{Pb}-\mathrm{Pb}$ ages in eucrite meteorites attributed to asteroid 4 Vesta (dubbed HEDs, for the howardite-eucrite-diogenite clan of achondrite meteorites collectively termed vestoids) are known to record a solid crust at 4563 Ma (Ireland \& Wlotzka 1992) and subsequent thermal modifications until ca. $4450 \mathrm{Ma}$ (Zhou et al. 2013; Hopkins et al. 2015). However, practically no such ages younger than ca. $4450 \mathrm{Ma}$ exist for any known meteorite class, including the vestoids. Younger ages for meteorite classes are instead largely confined to less retentive ${ }^{40-39} \mathrm{Ar}$ geochronology data sets (Bogard 1995), which generally display age continua from $\sim 4450 \mathrm{Ma}$ extending to about $3000 \mathrm{Ma}$, with occasional resetting events up to the present (e.g., Fernandes et al. 2013).

Despite these discrepancies, the lunar crater record continues its historical role as the standard reference for the nature of late accretion experienced by all solar system bodies (Öpik 1960; Shoemaker et al. 1962). Recent reanalyses, however, of purported ${ }^{40-39} \mathrm{Ar}$ age clusters at ca. $3950 \mathrm{Ma}$ from lunar rocks are inconclusive as to whether they were caused by an LHB or are the result of a monotonic decline (Boehnke \& Harrison 2016). As such, the problem of late accretion reduces to deciding for which period can the lunar crater record be used to interpret what primordial impact history connected with the ongoing accretion process after planet formation. Furthermore, how translatable is the lunar record to other solid surfaces of the solar system? Progress in deciphering this record would greatly enhance our understanding of the formation and evolution of the solar system as a whole and the thermal histories of the crusts of the terrestrial planets; the latter includes Earth's capacity to host a persistent biosphere from the earliest times (Mojzsis et al. 1996; Abramov \& Mojzsis 2009; Abramov et al. 2013).

\section{Role of Giant Planet Migration}

Notwithstanding debate over the existence of an LHB, a suite of dynamical models has been constructed to explain how a discrete and temporary surge in impactors could have transpired at some time in the formative stages of the early solar system (e.g., Gomes et al. 2005; Bottke et al. 2012; Marchi et al. 2012; Morbidelli et al. 2012, 2018; see Nesvorný et al. 2018, Clement et al. 2019). The common theme to all of these models is that they rely on a dynamical instability in the outer solar system. Indeed, support for orbital migration of the giant planets (Jupiter, Saturn, Uranus, and Neptune) in the first few hundred million years comes from several independent lines of evidence stemming from the orbital properties of both these planets themselves and analysis of small-body populations beyond Neptune (see Nesvorný 2018 for a recent review). For instance, Clement et al. (2019) present dynamical simulations that invoke a very early instability shortly after the dispersal of the protoplanetary disk. However, it is important to note that without temporal limits placed by geochronological data, caution is warranted in accepting any such model at face value. Giant planet migration is often, and erroneously, conflated with Grand Tack (Table 1). An early Grand Tack instability naturally reproduces the small mass of Mars (even if there are other models that can accomplish this strict constraint; e.g., Hansen 2009; Walsh et al. 2011; Izidoro et al. 2014; Brasser et al. 2017). Grand Tack is limited to the first million years or so, whereas giant planet migration (Nice model) occurs after that.
Subsequent to primary accretion, the giant planets are thought to have radially migrated because of their gravitational interactions with a massive external population of planetesimals (Fernandez \& Ip 1984). The migration of Neptune is chronicled in the orbital distribution of Pluto and the Kuiper Belt objects (Malhotra 1993, 1995). Dynamical models also show that giant planet migration requires planet-planet scattering (Thommes et al. 1999; Brasser et al. 2009; Morbidelli et al. 2010; Levison et al. 2011; Nesvorný \& Morbidelli 2012). Giant planet migration scenarios mutually predict that the inner solar system experienced a short but intense surge of cratering by comets sometime in the first 500 Myr (Gomes et al. 2005).

Two points must be emphasized about the nature of giant planet migration:

(1) the specific trigger for migration-and ensuing bombardment-is still mostly unknown; and

(2) its timing is inexplicit and could occur after a delay of a few million years to upward of several hundred million years (Levison et al. 2011).

Although debated, clear evidence for the role of outer solar system materials (i.e., comets) in this migration-induced bombardment is lacking on the Moon (Kring \& Cohen 2002; Strom et al. 2005; see Greenwood et al. 2011). Dynamical models of terrestrial planet formation all envisage a steady decline in bombardment rate once migration ceases (Neukum et al. 2001; Brasser et al. 2016). This expectation is entirely consistent with the crater chronologies cited above but is at odds with an LHB-like "spike" at any time after about $4400 \mathrm{Ma}$ (Hartmann et al. 2000). For instance, if giant planet migration commenced sometime after $4400 \mathrm{Ma}$ to give rise to a discrete LHB-type event, such an episode should be obvious as a distinct cluster of younger reset ages shared by all inner solar system bodies-including the Moon-in radiogenic systems relatively sensitive to thermal resetting, such as in ${ }^{40-39} \mathrm{Ar}$ and Rb-Sr (e.g., Bogard 1995; Fernandes et al. 2013). If, however, the migration commenced before $4400 \mathrm{Ma}$, endogenous crustal processes on geologically active worlds such as Earth and Mars are expected to have erased such evidence. This is not necessarily the case for a small, airless, old and cold body like asteroid 4 Vesta (Zhou et al. 2013), a fact that we use later in this analysis. The classical LHB case for a spike affecting the whole solar system after about $4100 \mathrm{Ma}$ (Ryder 2002) is further undermined by numerous documented pre-4100 Ma (and older) lunar ages (e.g., Petro \& Pieters 2006; Zellner 2017). These ages are surmised to reflect partial or complete resetting of high closure temperature mineral geochronological systems (e.g., Norman \& Nemchin 2014; Hopkins \& Mojzsis 2015; Kelly et al. 2018). A useful tool to interrogate early solar system bombardments is "closure temperature" (hereafter $T_{\mathrm{c}}$ ), defined as the temperature of a system at the time given by its apparent age (Dodson 1973; Reiners et al. 2005). We return to this point later.

Taken together, it should be evident that at least from the lunar record no firm conclusions can be drawn about the proposed mechanics of the early (before $4400 \mathrm{Ma}$ ) bombardment process, nor of the precise timing of giant planet migration that caused it. A new approach is warranted, and for this we turn to the asteroids. 


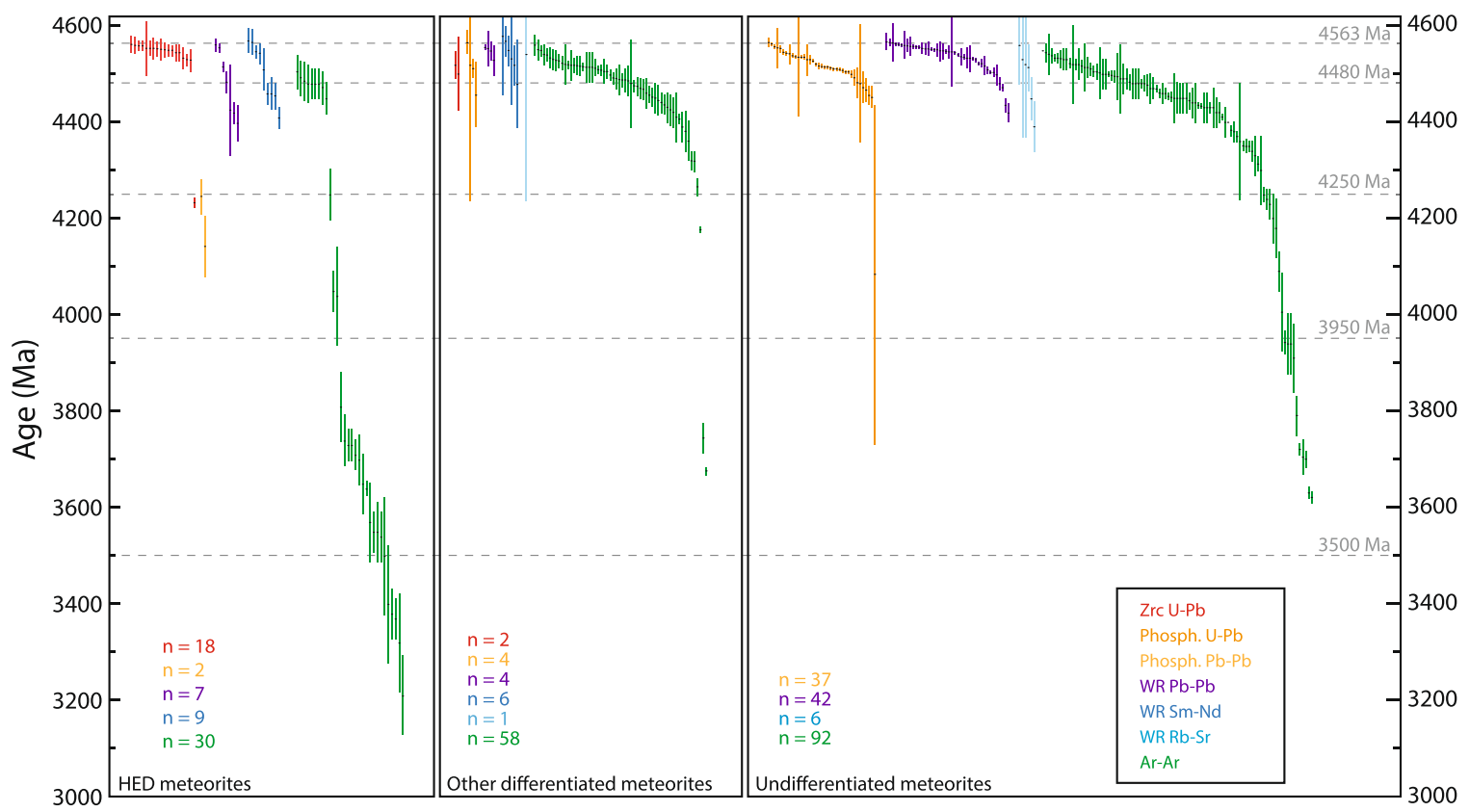

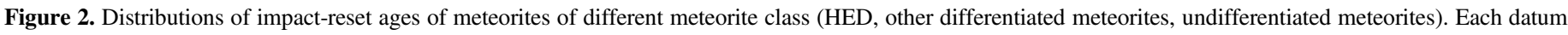

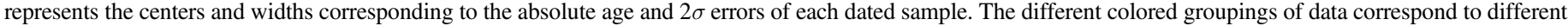

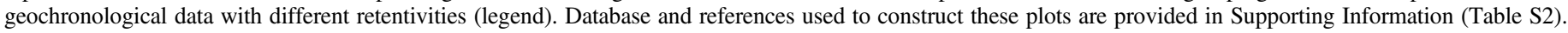

\section{Late Accretion and Asteroidal Meteorite Chronologies}

The diverse members of the asteroid belt (DeMeo \& Carry 2014) completed their formation before the inner planets and are thus witnesses to the solar system's earliest post-primary accretion history. We use compiled chronological data for asteroidal meteorites and compare different radiogenic systems with different $T_{\mathrm{c}}$ in different minerals and whole-rock samples to analyze this history. Our approach circumvents the debate between the monotonic decline and LHB "cataclysm" scenarios cited above because asteroidal meteorite ages are not anchored to the lunar record, and there is no dispute that they preserve a history that predates the Moon's own formation. Robust chronometers like $\mathrm{U}-\mathrm{Pb}$ in zircon from meteorites such as the brecciated eucrites - a subset of vestoids that have undergone substantial reworking through impacts-document thermal reset ages (Hopkins et al. 2015) for a system with substantially higher closure temperature $\left(T_{\mathrm{c}} \sim 1000^{\circ} \mathrm{C}\right.$; e.g., Reiners et al. 2015) than, for example, ${ }^{40-39} \mathrm{Ar}\left(250-450^{\circ} \mathrm{C}\right)$. Minerals that host radiogenic systems with intermediate $T_{\mathrm{c}}$, such as $\mathrm{Rb}-\mathrm{Sr}$ and $\mathrm{U}-\mathrm{Pb}$ in phosphate (e.g., basic $\mathrm{Ca}$ phosphate, or apatite) as well as whole-rock Sm-Nd geochronology (Borg et al. 2015), are expected to record ages of intermediate bombardment intensity. This approach bridges the gap between the highly retentive $\mathrm{U}-\mathrm{Pb}$ in zircon and the low retentivity of ${ }^{40-39} \mathrm{Ar}$ (Figure 2). Accordingly, $T_{\mathrm{c}}$ provides a clear theoretical basis for understanding meteorite mineral and whole-rock ages as cooling ages arising from the interplay between the kinetics of diffusion (or annealing), diffusant properties, crystal dimensions, and accumulation rates in cooling radio-isotopic systems from processes such as thermal effects of impacts.

Our thesis is simple: high- $T_{\mathrm{c}}$ highly retentive radiogenic systems will chiefly record older ages as opposed to the less retentive radiogenic systems. The most retentive systems such as $\mathrm{U}-\mathrm{Pb}$ in zircon (when available) will only ever record their own formation in a melt, or (rarely) the most energetic events caused either by higher impact velocities or by impacts from larger bodies, or both. The prediction is that $\mathrm{U}-\mathrm{Pb}$ in zircon registers ages of crust formation, or reset ages when the impact intensity is so high that the crust is molten and zircon crystallizes in melt sheets (see Wielicki et al. 2012). The ${ }^{40-39} \mathrm{Ar}$ system will only retain ages when the impact flux was low(er) and the solar system had dynamically relaxed.

An LHB-style surge in the impact spike from giant planet migration at any time in solar system history is expected to reset ages for the asteroid belt and appear as a cluster of ages in a variety of radiogenic systems, and not just in ${ }^{40-39} \mathrm{Ar}$.

To understand this further and explain the data in Figure 2, we now use our compendium from meteorites in Supporting Information (Table S2) with dynamical models to better constrain when the giant planets could have commenced their migration. This task requires that we model the temporal impact bombardment history onto the terrestrial planets by accounting for the major asteroids, leftover planetesimals of terrestrial planet formation, and comets arriving from the outer solar system. The outcomes of these models for late accretion, linked as they are to the geochronological constraints imposed herein, are then used to revise previous analyses of the thermal consequences of impact bombardments to the crusts of the planetary bodies of the inner solar system. Special focus is placed on the proclivity of the early Hadean Earth to support an emergent biosphere.

\section{Dynamics of Late Accretion Linked to Chronological Constraints}

The bombardment history of the terrestrial planets consists of three components: (i) dynamical evacuation of the (E-belt) asteroids, (ii) a contribution from leftover planetesimal material from terrestrial planet formation, and (iii) an influx of comets. In the following sections, we discuss the dynamical setup that determines the amount of late accretion onto the Moon and the terrestrial planets from E-belt asteroids and leftover 
Table 2

Impact Probabilities, Average Impact Velocities, and Mass Accreted from E-belt Material with the Terrestrial Planets, the Moon, 4 Vesta, and 1 Ceres

\begin{tabular}{lccc}
\hline \hline Planet & $\left\langle p_{\text {imp }}\right\rangle(\%)$ & $\left.\mathrm{s}^{-1}\right)$ & $\begin{array}{c}\left\langle v_{\text {imp }}\right\rangle(\mathrm{km} \\
\text { Expected Mass Striking the } \\
\text { Surface }(\mathrm{ppm})\end{array}$ \\
\hline Mercury & $0.90_{-0.03}^{+0.15}$ & $41_{-24}^{+27}$ & $71_{-56}^{+128}$ \\
Venus & $5.4_{-1.0}^{+0.7}$ & $26_{-14}^{+19}$ & $28_{-17}^{+39}$ \\
Earth & $5.5_{-1.0}^{+0.7}$ & $21_{-9}^{+14}$ & $24_{-15}^{+35}$ \\
Moon & $0.25_{-0.04}^{+0.03}$ & $18_{-12}^{+16}$ & $93_{-80}^{+154}$ \\
Mars & $2.2_{-0.2}^{+0.1}$ & $11_{-5}^{+11}$ & $94_{-67}^{+155}$ \\
4 Vesta & 0.016 & 9 & 1500 \\
1 Ceres & 0.0022 & 11 & 56 \\
\hline
\end{tabular}

planetesimals. The contribution from comets is discussed separately below owing to the fact that the dynamical model is much more involved.

\subsection{The E-belt}

The E-belt is a now-empty implied extension of the asteroid belt all the way to Mars that was evacuated by giant planet migration (Bottke et al. 2012). We compute the contribution to late accretion from the E-belt by running 32 dynamical $N$-body simulations of the evolution of this reservoir in the presence of the gravitational influence of the Sun and the eight planets on their current orbits. We also added asteroids 4 Vesta and 1 Ceres as massive bodies. Each simulation also has 512 massless asteroids. Our model employed the following initial conditions: the asteroids have a uniform distribution in semimajor axes $a$ from 1.7 to 2.1 au and a main-asteroid-beltlike Gaussian distribution in eccentricity $e$ and inclination $i$, with mean values of $0^{\circ} .15$ and 8.5 , respectively, and standard deviations of 0.07 and $7^{\circ}$, respectively. The other three angles (longitude of the ascending node, argument of perihelion, and mean anomaly) are chosen uniformly randomly between $0^{\circ}$ and $360^{\circ}$. The differences between each simulation are the different initial conditions of the planetesimals, even though these are statistically identical. We also ensured that initially none of the asteroids were on Mars-crossing orbits.

The simulations were run for $1 \mathrm{Gyr}$ of model time with SWIFT RMVS3 (Levison \& Duncan 1994) with a time step of 3.65 days. Planetesimals were removed once they were farther than 100 au from the Sun (whether bound or unbound) or when they collided with a planet or with the Sun. The impact probabilities were independently obtained from counting direct impacts onto the planets and from employing a post-processing procedure (Wetherill 1967) that computes the average impact probability. The latter is the only source to compute the impact probability with the Moon. We have previously tested this computational method against counting direct impacts with the planets, and we find that the two results match those in Bottke et al. (2012). The impact probabilities and average impact velocities for the terrestrial planets, 4 Vesta and 1 Ceres, are listed in Table 2. Analysis of the dynamics of late accretion to the terrestrial planets is forthcoming in future work.

We caution that the average impact velocity increases with time and that the values listed here are averaged over 1000 Myr. Consequently, a more refined temporal analysis of the flux exclusive to a particular epoch (e.g., limited to only 4450-4440 Ma) requires that we run many simulations to establish convergence. We also tabulate the expected amount
Table 3

Average Impact Probabilities and Impact Velocities of Leftover Planetesimals with the Terrestrial Planets, the Moon, 4 Vesta, and 1 Ceres

\begin{tabular}{lccc}
\hline \hline Planet & $\left\langle p_{\text {imp }}\right\rangle(\%)$ & $\begin{array}{c}\left\langle v_{\text {imp }}\right\rangle(\mathrm{km} \\
\left.\mathrm{s}^{-1}\right)\end{array}$ & $\begin{array}{c}\text { Expected Mass Striking the } \\
\text { Surface }(\mathrm{ppm})\end{array}$ \\
\hline Mercury & $5.2_{-1.5}^{+1.4}$ & $36_{-20}^{+24}$ & $919_{-734}^{+1280}$ \\
Venus & $17_{-3.1}^{+3.7}$ & $26_{-12}^{+15}$ & $170_{-130}^{+177}$ \\
Earth & $13_{-2.0}^{+2.5}$ & $22_{-9}^{+13}$ & $115_{-89}^{+130}$ \\
Moon & $0.59_{-0.20}^{+0.12}$ & $19_{-12}^{+15}$ & 250 (fixed) \\
Mars & $0.85_{-0.17}^{+0.13}$ & $15_{-9}^{+10}$ & $79_{-69}^{+139}$ \\
4 Vesta & $5.4 \times 10^{-4}$ & 13 & 534 \\
1 Ceres & $7.6 \times 10^{-4}$ & 13 & 710 \\
\hline
\end{tabular}

of mass striking the surfaces of Earth, the Moon, and Vesta from Monte Carlo impact experiments (Brasser et al. 2016) and their $2 \sigma$ uncertainties (see below). The uncertainties obtained from the assumed shallow size-frequency distribution are much larger than that in the impact probabilities. We further computed that the impact probability of objects from the main asteroid belt with the Moon is at least a factor of 30 lower than that of the E-belt, consistent with earlier estimates (Morbidelli et al. 2010). Since the two reservoirs are thought to initially have a similar mass (Bottke et al. 2005), the contribution from the main asteroid belt is thus insignificant in the first $1000 \mathrm{Myr}$ and henceforth neglected in our analysis.

\subsection{Leftover Planetesimals}

We have previously constrained the mass in leftover planetesimals from terrestrial planet formation (Brasser et al. 2016) based on the HSE abundance in the lunar mantle (Walker 2009). We then used that information to show that the purported "late veneer" (an early, albeit significant component of late accretion subsequent to core formation) to Earth and Mars consisted mostly of single, large impactors of dominantly enstatite or ordinary chondrite composition (Genda et al. 2017; Brasser et al. 2018; Woo et al. 2018, 2019). Because our analysis of leftover material requires that the Moon was present, we start our dynamical simulations at the time of the Moon-forming event, assigned here at $4500 \mathrm{Ma}$ (Barboni et al. 2017). We also possess a database of terrestrial planet formation simulations in the framework of the Grand Tack model (Walsh et al. 2011). Snapshots of the planetesimal population were taken $60 \mathrm{Myr}$ after the start of the simulations, which we approximately assign at $4500 \mathrm{Ma}$. We split the total number of planetesimals into 32 cases of 1074 massless planetesimals and added all the major planets, plus 4 Vesta and 1 Ceres, on their current orbits. Simulations were run with SWIFT RMVS3 for 500 Myr with a time step of 3.65 days with the same removal conditions described above for the E-belt simulations. Results for Earth, the Moon, and Vesta are provided in Table 3. Uncertainties are $2 \sigma$.

No direct record exists of how much mass was in these leftover planetesimals. Lunar HSE abundances show that the Moon accreted a further $0.025 \mathrm{wt}$.\% after its formation while it still had a magma ocean. Combined with the average impact probability with the Moon and employing a size-frequency distribution akin to that of the main asteroid belt suggests that the mass in leftover planetesimals at $4500 \mathrm{Ma}$ is approximately $4.7_{-3.7}^{+4.4} \times 10^{-3}$ Earth masses. To study the effect of the size-frequency distribution on 
the mass accreted by the planets, we rely on Monte Carlo impact experiments.

We recently combined $N$-body and Monte Carlo simulations to establish the nature of late accretion onto the terrestrial planets and the likely total mass accreted by Earth and Mars when calibrated to lunar HSE abundances (Brasser \& Mojzsis 2017). Due to the assumed shallow size-frequency distribution of the impactors, the ratio of the total mass accreted by Earth versus that of the Moon is generally much higher than the ratio of their gravitational cross sections (Bottke et al. 2010). The accreted mass is dominated by stochastic accretion from a few large impactors (Tremaine \& Dones 1993). We presupposed that the bodies in the simulations had minimum diameters of 1 $\mathrm{km}$, a maximum diameter of $2000 \mathrm{~km}$, and a bulk density of $2500 \mathrm{~kg} \mathrm{~m}^{-3}$ for all objects regardless of their size. Two cases are tested: we either presume the same shallow size-frequency distribution as that of the main asteroid belt, or with a changeover to a steeper cumulative slope of 4.5 at a projectile diameter of $50 \mathrm{~km}$ (Johnson et al. 2016). For further details of the method we refer the reader to Brasser et al. (2016). The expected values of material accreted by Earth and Mars are much lower than their actual values of $0.7 \mathrm{wt} \%$ and $0.8 \mathrm{wt} \%$, respectively (Day et al. 2016); these outcomes are discussed extensively in Hartmann et al. (2000). We did not include 4 Vesta and 1 Ceres in the Monte Carlo code because they are not expected to be struck by large bodies, so the listed values are estimates obtained from the dynamical simulations.

\subsection{Comets}

The cometary impact flux onto the terrestrial planets is caused by the late migration of the giant planets. By late we mean well after the gas of the protosolar nebula has dissipated (Table 1); we assume that the gas disk is dispersed by ca. 4563 Ma based on the youngest chondrule ages (e.g., Morris et al. 2015; Bollard et al. 2017). Late giant planet migration is caused by the scattering of distant planetesimals beyond Neptune and by mutual scattering among the giant planets (e.g., Tsiganis et al. 2005).

We computed the cometary flux onto the terrestrial planets in three sequential steps. Step 1: Find the best initial conditions for the giant planets and the planetesimal disk that ultimately has the highest probability of reproducing the current architecture of the terrestrial planets. Step 2: Compute the fraction of comets from the trans-Neptunian disk that venture closer than 1.7 au from the Sun as a proxy for objects that reach the terrestrial planets. Step 3: Compute the impact probability of these comets with the terrestrial planets. Step 4: Apply Monte Carlo impact simulations to the comets to compute the amount of material that strikes Earth and the Moon.

These steps are now explained in detail.

Step 1: Initial conditions. The evolution of the giant planets during late migration is chaotic (Tsiganis et al. 2005), and the probability of the planets ending up near their current orbits is low: 5\% (Brasser \& Lee 2015; Nesvorný 2015a). The aim is to increase that probability by varying the initial conditions and decide which combination of three input parameters best reproduces the current configuration of the giant planets. Here we follow the procedure outlined in Wong et al. (2019).

The initial conditions employed here are identical to a loose five-planet configuration (Nesvorný 2011) because this configuration best reproduces the current orbital structure of the Kuiper Belt (Nesvorný 2015b). To start, we assume that there are five giant planets in the quadruple resonance $3: 2,3: 2,2: 1$, $3: 2$. The initial conditions for this configuration were provided by D. Nesvorný (2019, personal communication), but we allowed the initial semimajor axis of Jupiter to vary (first free parameter); the initial semimajor axes of the other planets were computed from their resonant locations. Variations in the initial conditions within each set were obtained by giving a uniformly random deviation of $10^{-6}$ au to the position vector of each planetesimal.

In our simulations, the planetesimal disk consisted of 3000 objects. The surface density of the planetesimal disk scales with heliocentric distance as $\Sigma \propto r^{-1}$. The total disk mass is the second free parameter. The inner edge of the disk is located 1 au outward from the outermost ice giant; the outer edge of the disk is our third free parameter.

We run many sets of 128 simulations each wherein the three free parameters are permutated. We make use of the symplectic integrator SyMBA (Duncan et al. 1998). Planets and planetesimals were removed once they were farther than 1000 au from the Sun (whether bound or unbound) or when they collided with a planet or ventured closer than 0.5 au to the Sun. We evolve every simulation using a time step of $0.4 \mathrm{yr}$ to $500 \mathrm{Myr}$ or until fewer than four planets remain, whichever occurs first. The migration evolution and final semimajor axes and eccentricities for the giant planets are provided in Figure 3. We show cases for a planetesimal disk of 18 (red symbols) and 19 Earth masses (blue symbols). The black circles and error bars denote the current semimajor axes and eccentricities and the extent of their secular variation. The arrows indicate the direction and amount of migration. We conclude that the combination of parameters that yields the highest probability of reproducing the current solar system according to the criteria of Nesvorný (2015b) has Jupiter initially at 5.6 au, a disk mass of 18 Earth masses, and an outer edge at 27 au. These parameters are used as input for Step 2.

Step 2. Compute the fraction of comets that enter the inner solar system. In order to calculate the impact probability of comets onto the Moon and the terrestrial planets, we use a piecewise approach and split the work across two sets of simulations.

The model used the best initial conditions determined in Step 1 to run one set of 512 simulations with the same initial configuration of the planets, disk mass, and disk outer edge, but now comets were removed from the simulation when they ventured closer than 1.7 au from the Sun. The vectors and the time of crossing $1.7 \mathrm{au}$ are stored for the next step.

We find that $22 \%$ of all comets from the trans-Neptunian disk crossed the 1.7 au barrier, or the equivalent of ca. $4 M_{\oplus}$. The outcome of this experiment is used in step 3.

Step 3. Calculate the impact probability of the comets with the terrestrial planets. All the comets that crossed the $1.7 \mathrm{au}$ barrier were then integrated for $10 \mathrm{Myr}$ in simulations that included all eight planets on their current orbits, as well as 4 Vesta and 1 Ceres. This duration is about two orders of magnitude longer than the average dynamical lifetime of a Jupiter-family comet (Brasser \& Morbidelli 2013). The comets were introduced into the simulation with the position and velocity when they crossed the barrier from Step 2. All runs were performed with SWIFT RMVS3 with the same time step and removal criteria as for the leftover planetesimals.

The comets are dynamically controlled by Jupiter, and consequently they only ever spend little time in the inner solar 


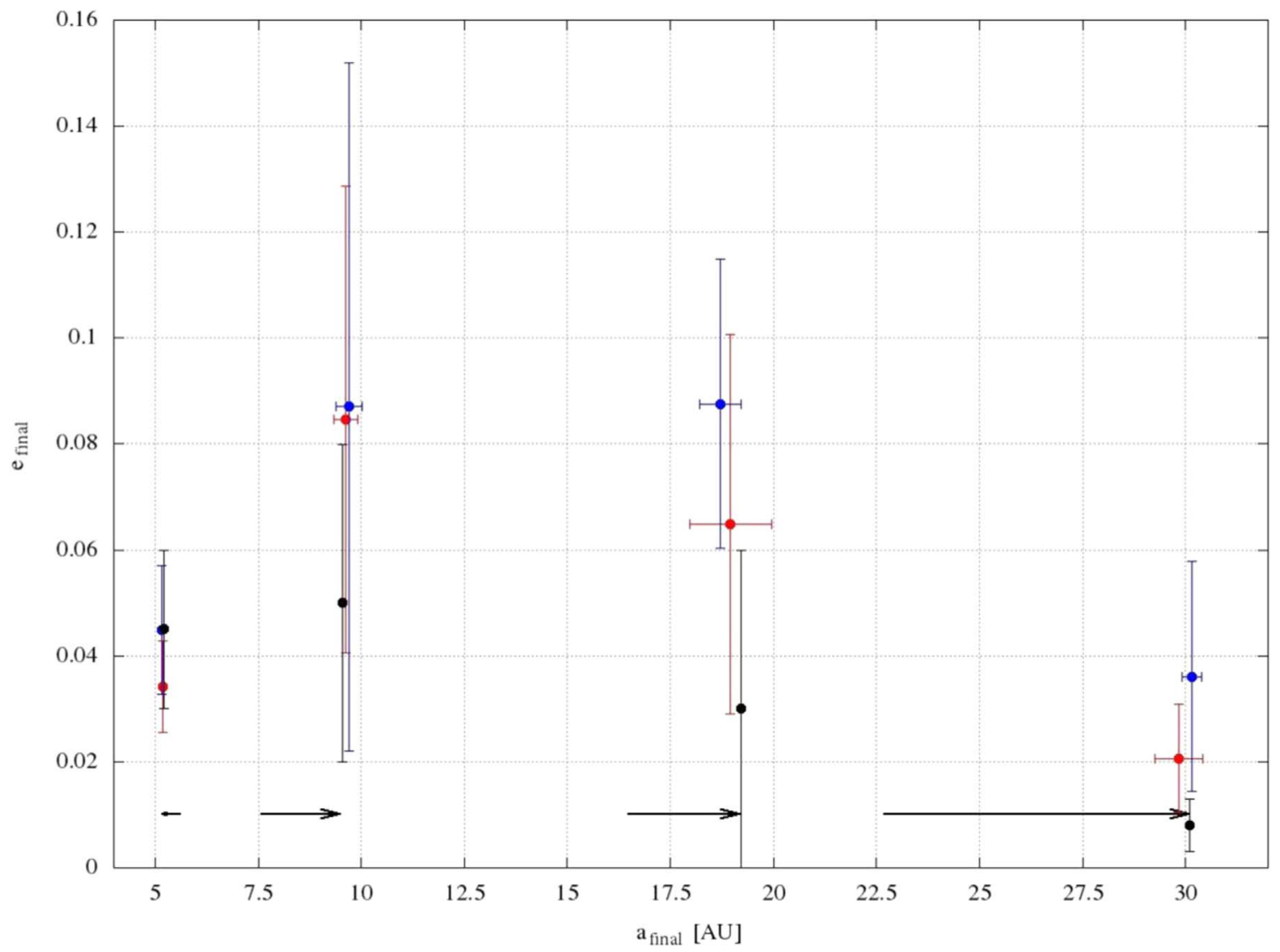

Figure 3. Final semimajor axes and eccentricities of giant planets after migration. Arrows indicate amount and direction.

system (Levison \& Duncan 1997; Di Sisto et al. 2009). We therefore do not record any physical impacts on the terrestrial planets and resort to an averaging procedure (Wetherill 1967) that computes the impact probability and therefore the total accreted mass. Table 4 shows the cometary impact probabilities with Earth, the Moon, and 4 Vesta when taking into account the $22 \%$ probability of the comets venturing into the inner solar system from Step 2 herein. We also list the average impact velocity and the expected amount of mass in comets that strikes the surface of each of these bodies.

Step 4: Monte Carlo impact experiments. We apply the same Monte Carlo experiments to the comets to compute the amount of material that impacts Earth and the Moon. The simplest size-frequency distribution that matches the Kuiper Belt is used, with a change in slope at a diameter of ca. $60 \mathrm{~km}$ and cumulative slope indices of 4.8 and 1.9, respectively, at the high and low ends (Fraser \& Kavelaars 2009). Since the distribution exhibits a knee at diameters of $60 \mathrm{~km}$, most of the mass in the belt resides in objects of approximately this size, and thus we expect that the ratio of accreted masses between the planets is approximately equal to their gravitational cross sections. We assumed a bulk density of $1400 \mathrm{~kg} \mathrm{~m}^{-3}(\sim 2 \times$ computed average comet density; Peale 1989). Outcomes of these analyses are shown in Figures 4-6.

To synopsize this section, we describe how bombardment to the terrestrial planets consists of comets, leftovers of accretion, and E-Belt asteroids. The results of our analyses for the rates of impacts from these three sources to Earth and the Moon are reported in Figure 5.
Table 4

Impact Probabilities and Average Impact Velocities of Cometary Material with the Terrestrial Planets, Moon, 4 Vesta, and 1 Ceres

\begin{tabular}{lccc}
\hline \hline Planet & $\left\langle p_{\text {imp }}\right\rangle\left(\times 10^{-6}\right)$ & $\begin{array}{c}\left\langle v_{\text {imp }}\right\rangle(\mathrm{km} \\
\left.\mathrm{s}^{-1}\right)\end{array}$ & $\begin{array}{c}\text { Expected Mass Striking the } \\
\text { Surface }(\mathrm{ppm})\end{array}$ \\
\hline Mercury & $0.13_{-0.08}^{+0.22}$ & $34_{-5}^{+10}$ & $42_{-13}^{+14}$ \\
Venus & $1.9_{-1.3}^{+2.9}$ & $26_{-2.5}^{+3.8}$ & $42_{-7}^{+7}$ \\
Earth & $3.1_{-2.8}^{+3.3}$ & $22_{-1.7}^{+2.9}$ & $57_{-8}^{+8}$ \\
Moon & $0.16_{-0.08}^{+0.20}$ & $20_{-1.9}^{+3.7}$ & $239_{-70}^{+64}$ \\
Mars & $2.5_{-1.2}^{+3.3}$ & $13_{-0.6}^{+1.9}$ & $409_{-42}^{+59}$ \\
4 Vesta & 0.0093 & 11 & 3900 \\
1 Ceres & 0.025 & 11 & 2900 \\
\hline
\end{tabular}

Note. Monte Carlo simulations were only run for Earth, the Moon, and Mars.

\section{Geo- and Biophysical Consequences of Late Accretion to Hadean Earth}

Bombardment that accompanied late accretion strongly modulated the initial physical, chemical, and (pre-)biological state of the Hadean Earth. Here we assess how our new massproduction functions for late accretion affected the young Earth's crust using improved impact parameters for comets, leftovers of accretion, and E-belt asteroids.

Our new global thermal cratering analysis consists of (i) a stochastic cratering model that populates a cuboid representing the Hadean Earth's surface with craters using the massproduction functions derived from the results of our new dynamical models; (ii) analytical expressions that calculate a temperature field for each crater (Abramov \& Mojzsis 2009; 
Moon

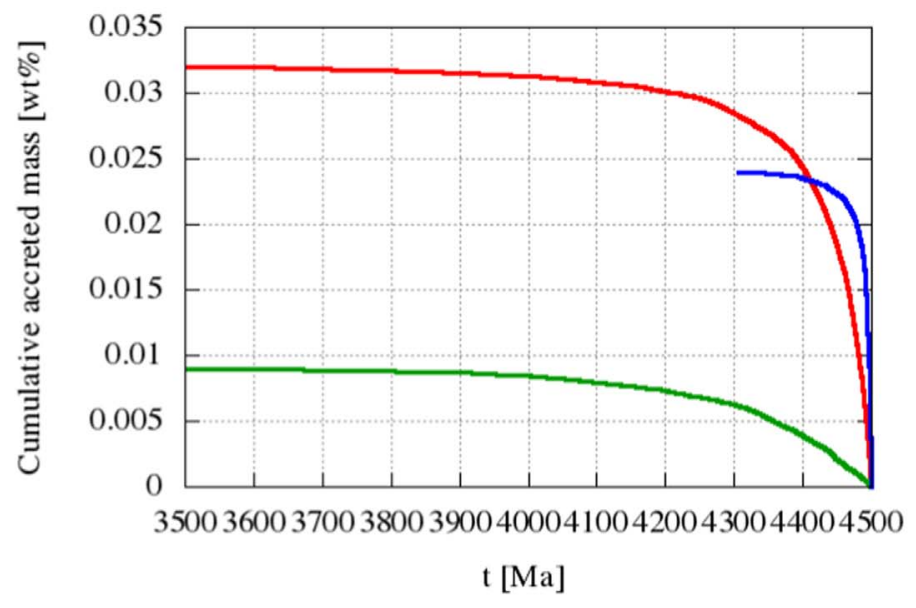

Mars

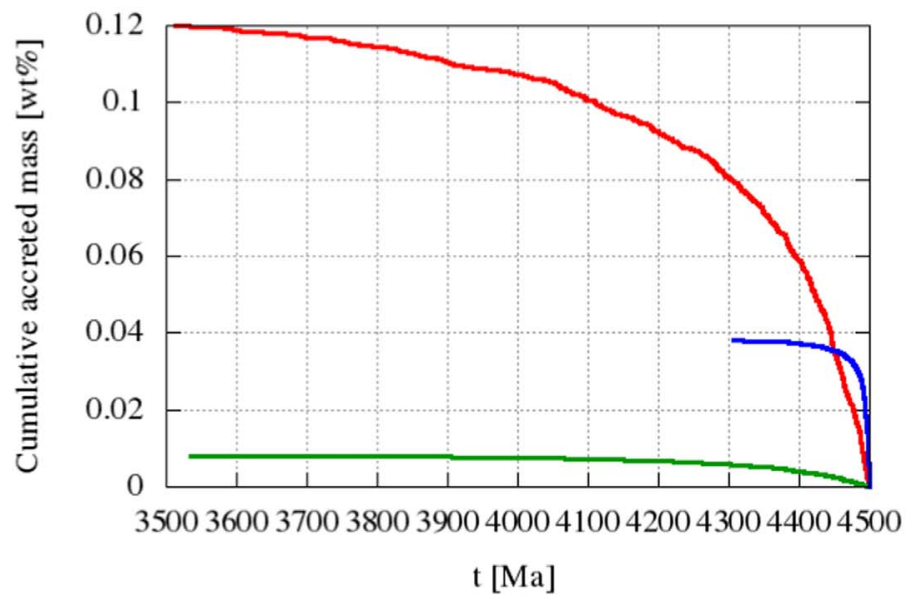

Earth

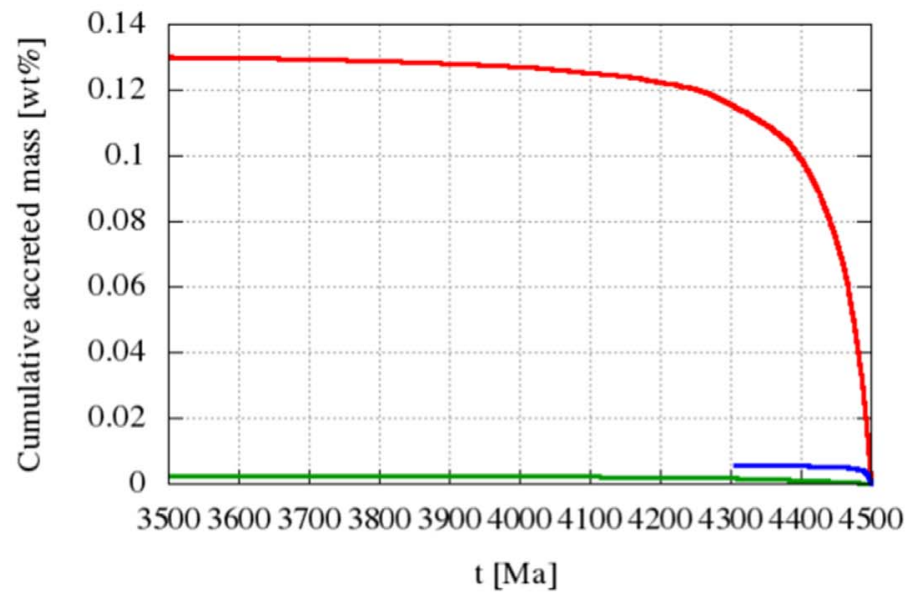

Venus

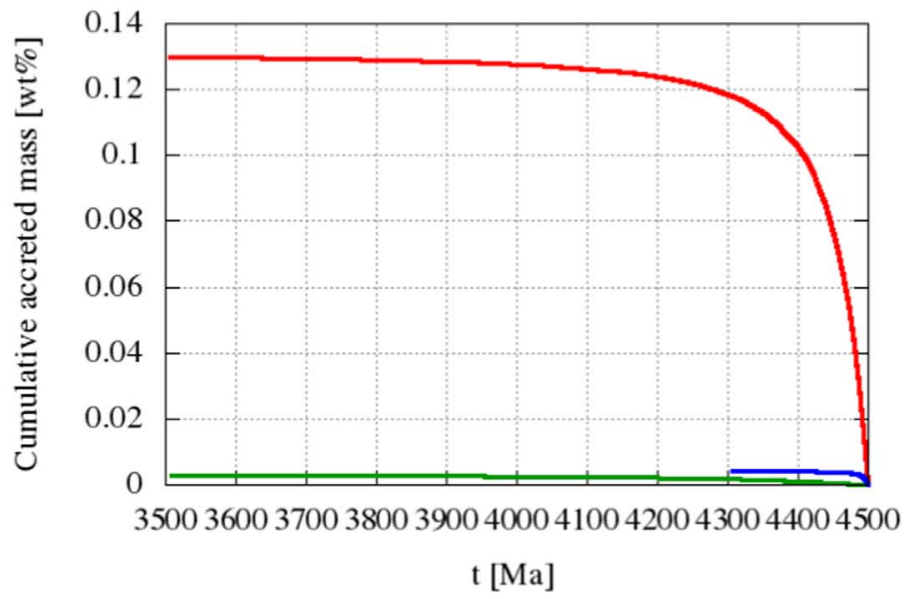

Figure 4. Accretion profiles for the Moon, Earth, Mars, and Venus assuming that the giant planet instability occurred at 4.5 Ga. This plot supposes that all impactors have the same mass. Red is for leftover planetesimals, blue is for comets, and green is for the E-belt.

Abramov et al. 2013); (iii) a three-dimensional thermal model of the terrestrial lithosphere, where craters are allowed to cool by conduction and radiation; and (iv) instantaneous plus cumulative melt production, as well as defined thermal volumes of the crust as a function of time (Kieffer \& Simonds 1980; Pierazzo \& Melosh 2000). The model output reports the amount of melting in the lithosphere, both instantaneous and cumulative, as well as thermal volumes in the top $4 \mathrm{~km}$ of the lithosphere (surface to depth) at any point in time corresponding to temperature fields known for various microbial communities in the crust (mesophile, thermophile, hyperthermophile).

\subsection{Late Accretion to the Hadean Earth Surface}

The outputs from simulations of impact bombardment experienced by Earth between 4500 and $4000 \mathrm{Myr}$ are shown in Figure 7. In our baseline model, the lithosphere thickness does not change from 4500 to $4000 \mathrm{Ma}$ and remains $24 \mathrm{~km}$ thick (solid lines in Figure 7(a)). We also ran a model where we start with a magma ocean at $4.5 \mathrm{Ga}$ and have lithospheric thickness increase with time due to radiative cooling (dashed line in Figure 7(a)). Mean surface temperature and geothermal gradient were assumed to be $20^{\circ} \mathrm{C}$ and $70^{\circ} \mathrm{C} \mathrm{km}^{-1}$, respectively, and a basaltic composition was assigned for the initial lithosphere. Total delivered mass was $7.76 \times 10^{21} \mathrm{~kg}$, equivalent to $0.13 \%$ of Earth's mass (Supplementary video file 1 ), slightly more than the baseline model, although $0.065 \mathrm{wt} \%$ delivered mass was also tested. The size-frequency distribution of these late accretion impacts was approximated using the size-frequency distribution of the main asteroid belt. The velocity distribution of the impactors, as well as the decline of impact flux with time, was derived from dynamical modeling as described herein. Impactor density of these rocky bodies was assumed to be $3000 \mathrm{~kg} \mathrm{~m}^{-3}$, and impact angle of each impactor was stochastically generated from a Gaussian distribution centered at $45^{\circ}$. Some of our simulations began with a magma ocean scenario (Supplementary video file 2). We explored this scenario owing to the fact that we deem it to be a likely consequence of the largest post-Moon formation impactors in late accretion implicated, for example, in Earth's postulated late veneer (e.g., Genda et al. 2017). Based on previous work by us (Abramov \& Mojzsis 2009) and others (Chyba 1990; Marchi et al. 2014), we estimate that a minimum total delivered mass of $9.31 \times 10^{21} \mathrm{~kg}$ of accreted material is required to melt the terrestrial lithosphere at any particular point in time. Assuming asteroid 1 Ceres's density, this corresponds to an object of approximately $1200 \mathrm{~km}$ in diameter (about the size of Pluto's moon, Charon) striking Earth to melt the crust. This condition is also satisfied by a multitude of smaller objects, but we 
Earth
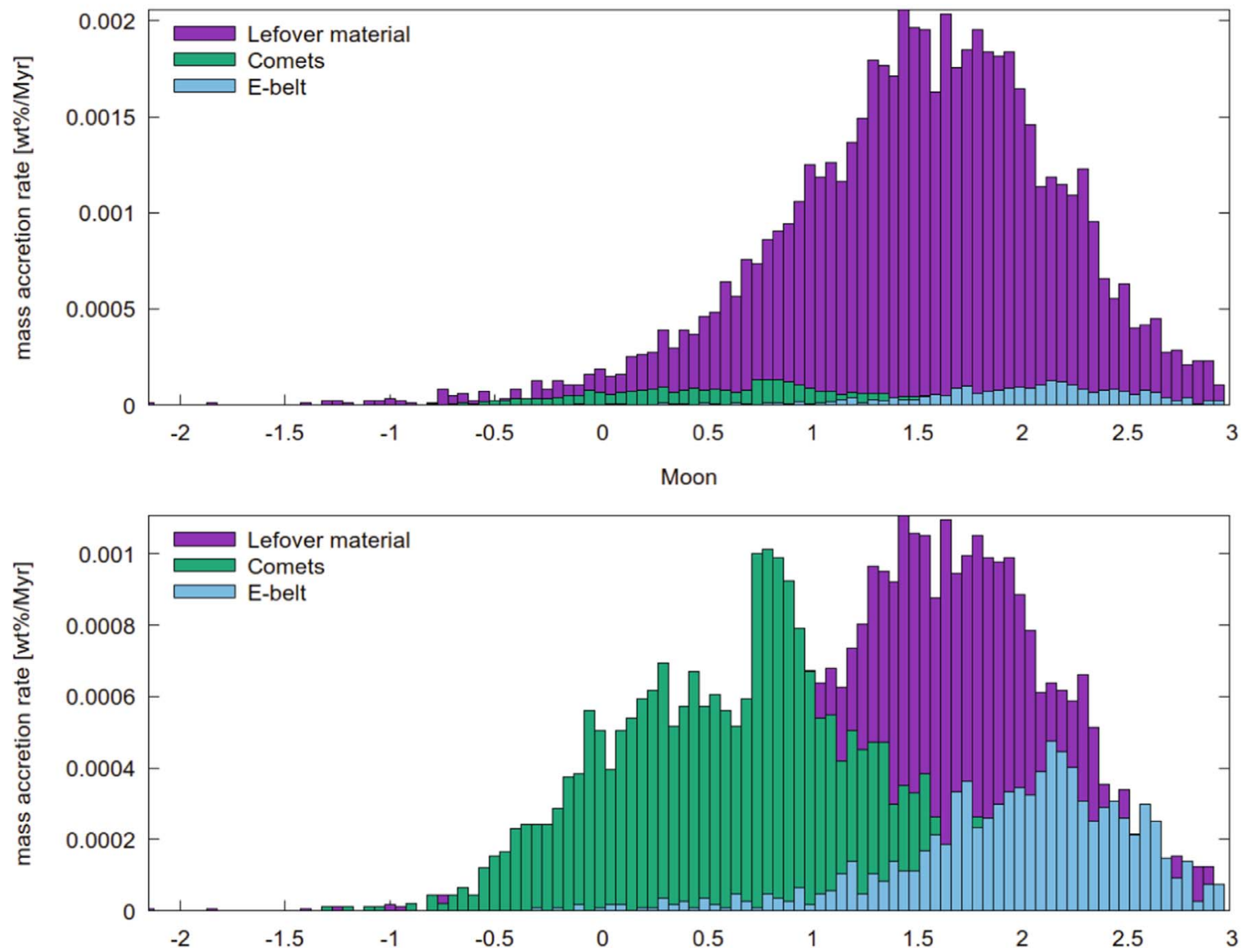

Figure 5. Impact rates onto Earth and the Moon from comets, leftover planetesimals, and the E-belt expressed as mass accretion rate in weight percent/Myr on target (Earth, Moon) vs. time expressed in $\log /$ Myr.

consider that scenario to be less likely than the single-strike case (see Brasser et al. 2016 for this argument). At a 13\% impact probability with Earth, the total mass in leftover planetesimals required to account for melting the terrestrial lithosphere is 0.011 Earth masses at $4500 \mathrm{Ma}$; this is well within acceptable parameter space (Brasser et al. 2016).

\subsection{Asteroid 4 Vesta as an Archive}

Our dynamical simulations also indicate that asteroid Vesta is struck by $0.39 \mathrm{wt} \%$ of cometary material (Table 4), which translates to $10^{18} \mathrm{~kg}$; the equivalent diameter of an object of that mass is about $110 \mathrm{~km}$. The typical mass of the largest object to impact Vesta for the size-frequency distribution used here is $\sim 1 / 3$ of the total mass (Tremaine \& Dones 1993). This suggests that the diameter of the largest comet to have struck this asteroid is about $76 \mathrm{~km}$, or about one-eighth of the diameter of Vesta itself. If it was struck by an object twice as large, we expect that such an impact could have mechanically disrupted the asteroid. Using a typical impact velocity of $11 \mathrm{~km}$, impact with an object of diameter $76 \mathrm{~km}$ would raise the temperature of about $2.5 \times 10^{5} \mathrm{~km}^{3}$ of Vesta's crust (or about $0.35 \%$ of its total volume) above $1100^{\circ} \mathrm{C}$ (see Figure 8 in Hopkins et al. 2015). Our estimate of the asteroid's crustal resetting should be considered as a lower limit since twice as much mass will strike its crust in smaller projectiles. If we naively assume that the heat spreads evenly across the asteroid's surface, this amount of volume would yield a layer
$286 \mathrm{~m}$ deep available to thermally reset any radiogenic ages in that volume. Therefore, if the cometary bombardment occurred in an LHB-like scenario, we would expect that the brecciated vestoid meteorites (eucrites) should show substantial late $\mathrm{U}-\mathrm{Pb}$ reset ages in zircon around 4100 or 3950 Ma. They do not.

\section{Results and Discussion}

Our compilation of asteroidal meteorite age data does not show a common LHB-like spike pattern near $3950 \mathrm{Ma}$, and we can rule out a specific solar-system-wide bombardment at that time caused by giant planet migration. Anticipated LHB patterns do not exist at 4100-4200 Ma either, nor are they present back to ca. $4400 \mathrm{Ma}$. Highly retentive radiogenic $\mathrm{U}-\mathrm{Pb}$ and $\mathrm{Pb}-\mathrm{Pb}$ ages older than $4530 \mathrm{Ma}$ - variably preserved in ordinary chondrites, vestoids, and other meteorite groups in Figure 2-are attributable to parent body formation ages (Ireland \& Wlotzka 1992; Bogard 1995). Crustal processes inherent to these small bodies that could reset ages after $4530 \mathrm{Ma}$ are precluded owing to the demise of the principle short-lived radioactive heat source to the early solar system ${ }^{26} \mathrm{Al}$ (e.g., Lichtenberg et al. 2016) and the dearth of other radioactive heat sources with sufficient power output (e.g.. $\left.{ }^{60} \mathrm{Fe},{ }^{244} \mathrm{Pu}\right)$. Hence, our preferred interpretation of ages substantially younger than 4530 Ma for any asteroidal meteorite is that they document impact-induced thermal resets past a particular $T_{\mathrm{c}}$ of a radiogenic system from a protracted decline of late accretion. 
E-belt
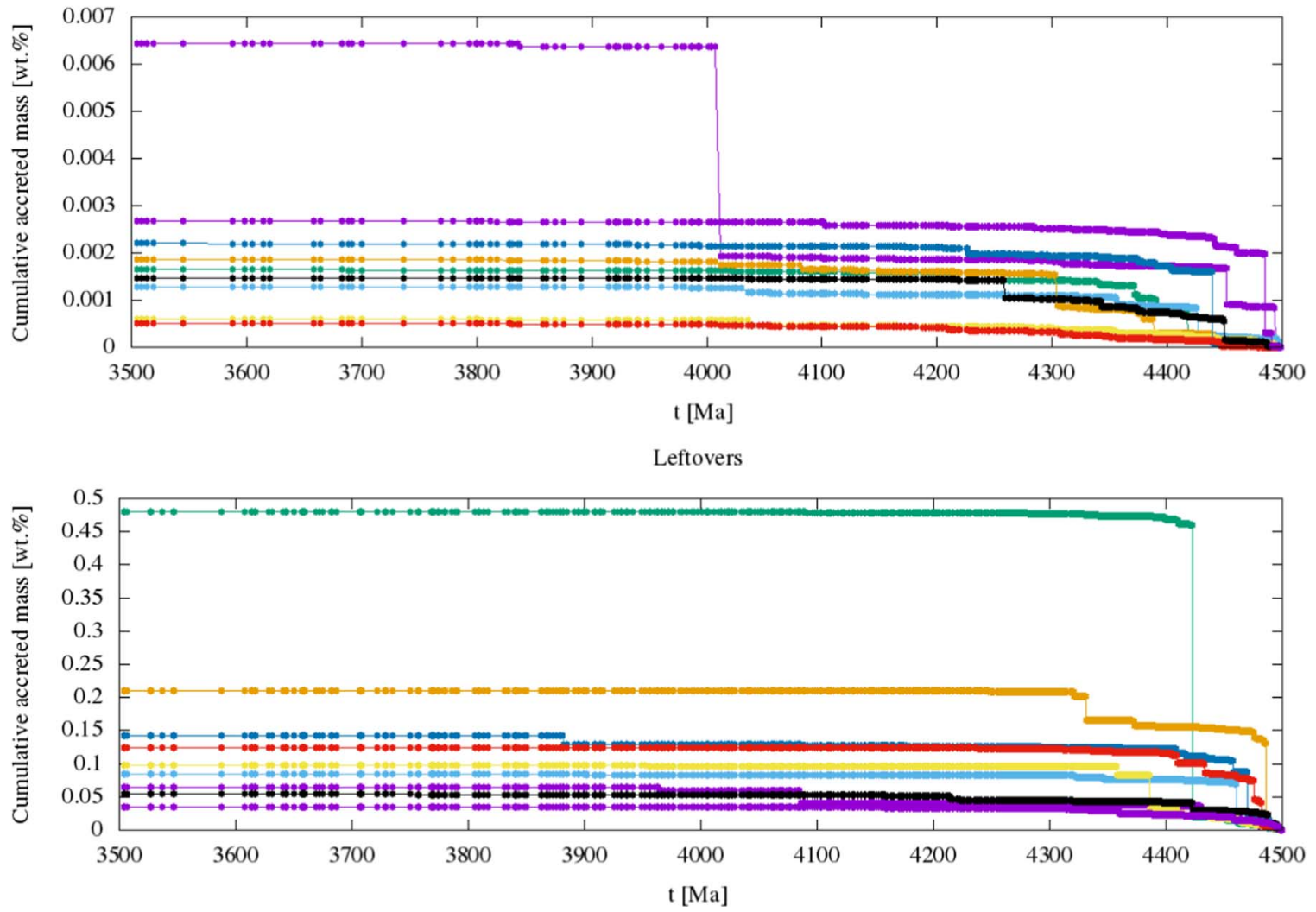

Figure 6. Late accretion onto Earth from the E-belt (top) and leftover material (bottom) taking the size-frequency distribution of the impactors into account.

Intriguingly, a number of meteorite classes display patterns of $\mathrm{U}-\mathrm{Pb}$ and $\mathrm{Pb}-\mathrm{Pb}$ in zircon and apatite age resets that end at about $4450 \mathrm{Ma}$. The whole-rock $\mathrm{Pb}-\mathrm{Pb}$ and $\mathrm{Sm}-\mathrm{Nd}$ systems, with intermediate $T_{\mathrm{c}}$ of $600^{\circ} \mathrm{C}$ (below that of $\mathrm{U}-\mathrm{Pb}$ in zircon, but above ${ }^{40-39} \mathrm{Ar}$ ), terminate later, at around $4400 \mathrm{Ma}$. We find that ${ }^{40-39} \mathrm{Ar}$ ages tend to pick up near $4480 \mathrm{Ma}$, roughly where $\mathrm{U}-\mathrm{Pb}, \mathrm{Pb}-\mathrm{Pb}$, and $\mathrm{Sm}-\mathrm{Nd}$ ages leave off, with little overlap between the groups. Argon data also show a continuum in ages extending from about 4480 to $3000 \mathrm{Ma}$, and younger. We view this behavior of sequential termination of ages in minerals with decreasing $T_{\mathrm{c}}$ as the expected result of a long monotonic decline of leftover debris of planet formation and the product of occasional inter-asteroid collisions (Bottke et al. 2005). The result is in agreement with crater counting statistics for the Moon and generally comports with the meta-analysis of compiled lunar ${ }^{40-39}$ Ar data by Boehnke \& Harrison (2016). It is decidedly at odds, however, with any bombardment model that calls for cataclysmic spikes in impact activity at any time in solar system history since about $4450 \mathrm{Ma}$.

It may also be the case that the relative rarity of resolved ${ }^{40-39} \mathrm{Ar}$ ages older than ca. $4450 \mathrm{Ma}$ for the asteroidal meteorites results from the "Stonewall Effect" (Hartmann et al. 2000; Hartmann 2003), in which a history of earlier thermal events becomes masked. In the Stonewall scenario, the last impacts energetic enough to reset ages are recorded, and earlier incidents tend to become so severely overprinted that they become effectively obliterated. The near absence of reset ages younger than $4450 \mathrm{Ma}$ in, for example, robust $\mathrm{U}-\mathrm{Pb}$ and $\mathrm{Pb}-\mathrm{Pb}$ chronologies for asteroidal meteorites also agrees with trends of declining bombardment intensity in line with our new dynamical models. Collectively, meteorite age distributions viewed through the lens of dynamical studies presented herein show that giant planet migration commenced around $4480 \mathrm{Ma}$, since the ensuing cometary bombardment records no cluster of ages at any time after.

\subsection{Giant Planet Migration Occurred around $4480 \mathrm{Ma}$}

We expect that a signature for giant planet migration should also be evident in traces of a brief but intense cometary input to the moons and planets as proposed by Gomes et al. (2005) and shown in Figure 3. For example, lunar hydrogen isotope $(\mathrm{D} / \mathrm{H})$ values suggest that the Moon accreted some cometary material after it formed (Greenwood et al. 2011, 2018). Xenon isotopes on both Earth and the Moon hint that these bodies acquired some part of their noble gas inventories from comets (Bekaert et al. 2017; Marty et al. 2017); it has also been postulated that some Xe in the Moon was inherited from Earth in a "synestia" scenario (Lock et al. 2018). It seems more likely that cometary Xe was delivered to the terrestrial planets after the Moonforming event. This is because both the lunar Xe shows evidence for it and, unless the target proto-Earth was dry at the time, the giant impact that formed the Moon is expected to have stripped Earth of much of its primary atmosphere, including the noble gases (Genda \& Abe 2005; Schlichting \& Mukhopadhyay 2018). We view these combined lines of evidence as a means to narrow the time interval during which the giant planets migrated to soon after Moon formation, and perhaps coincident with it.

Further constraints arise from lunar origin models and dynamical history of binary objects in the outer solar system. The time for the proposed giant impact that formed the Moon is 


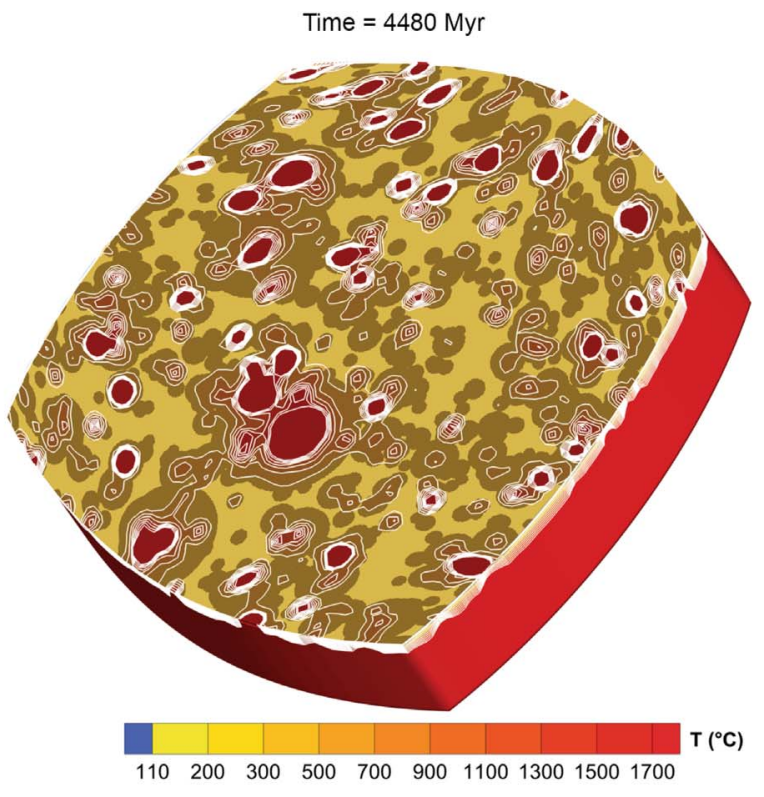

Earth, $0.13 w t \%$ delivered mass, dashed line indicates magma ocean

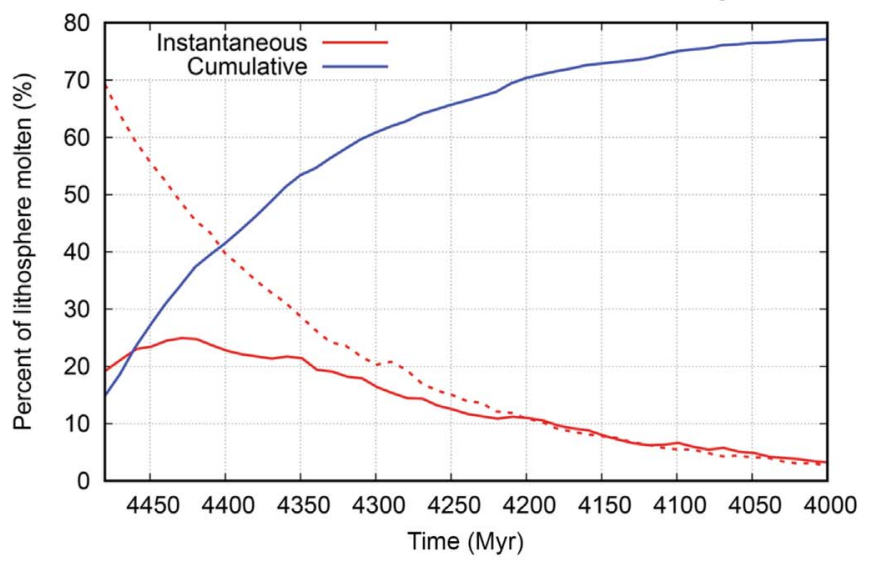

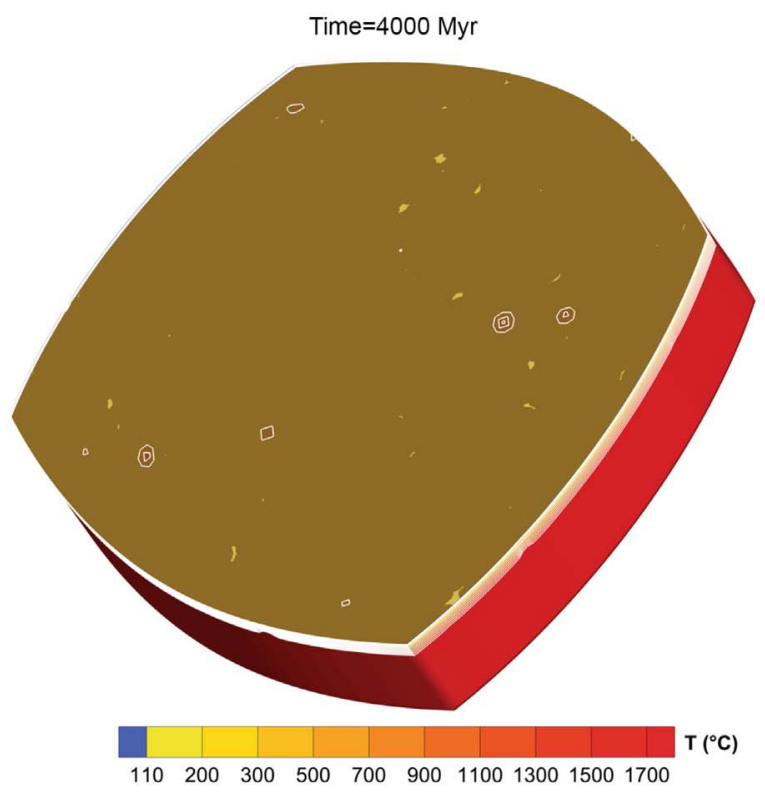

Earth, 0.13 wt\% delivered mass, dashed line indicated magma ocean

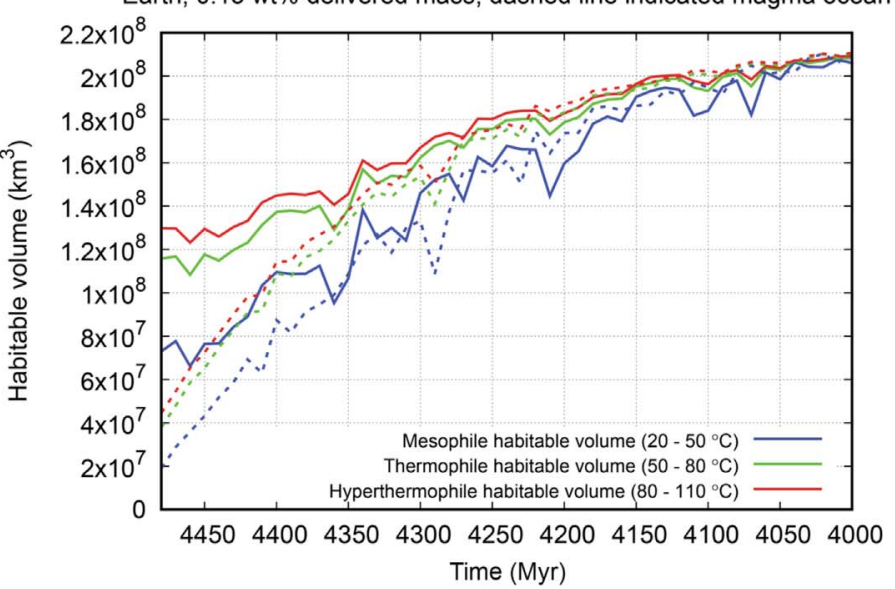

Figure 7. Three-dimensional thermal models representing the entire volume of Hadean Earth's lithosphere as a full wraparound cuboid at various times during late accretion (a) beginning at $4480 \mathrm{Myr}$ and (b) concluding at $4000 \mathrm{Myr}$ ago. Only impactors larger than $1 \mathrm{~km}$ in diameter are included in this representation; our baseline model was $0.13 \mathrm{wt} . \%$ of delivered mass to Earth over the course of late accretion. The top surface in this model rendering indicates temperatures at a depth of $4 \mathrm{~km}$, and the bottom surface indicates temperatures at a depth of $140 \mathrm{~km}$. Dark areas indicate crater imprints. The abrupt boundary transition to $1700^{\circ} \mathrm{C}$ is due to the chosen color scheme, whereby all temperatures higher than $1700^{\circ} \mathrm{C}$ appear red. This is to emphasize the approximate lithosphere/asthenosphere boundary. (c) Immediate thermal effects of impacts on the lithosphere. Percent of Earth's lithosphere to experience melting at any given time during late accretion. The solid line represents the baseline model, and the dashed line represents the model with an initial magma ocean condition. Lithospheric thickness has no significant effect on these results. (d) Thermal states of crustal volumes in hydrothermal environments. Solid lines represent the baseline model, and the dashed lines represents the model with an initial magma ocean condition. Mesophile $\left(20-50^{\circ} \mathrm{C}\right.$, blue), thermophile $\left(50-80^{\circ} \mathrm{C}\right.$, green), and hyperthermophile $\left(80-110^{\circ} \mathrm{C}\right.$, red) thermal tolerance volumes are reported for 500 Myr of bombardment and resultant active hydrothermal environments. Data to support these plots are provided in Supporting Information (Table S3).

(The data used to create this figure are available).

(An animation of this figure is available.)

contested but may be close to 4510 Ma (Barboni et al. 2017; see Boyet \& Carlson 2007; Borg et al. 2011; Connelly \& Bizzarro 2016). Our dynamical tests show that the cometary bombardment should have endured for about 30 Myr. Such an intense but short-lived flux of comets that began at $4480 \mathrm{Ma}$ and ended by 4450 Ma would be incapable of further wholesale crustal destruction and concomitant resetting of highly retentive ages in any sampled solar system object, including lunar rocks (Nemchin et al. 2009; Norman \& Nemchin 2014; Hopkins \& Mojzsis 2015; Kelly et al. 2018) and vestoid meteorites (Hopkins et al. 2015).
The ostensible timing of coincident impacts of large "late veneer"-scale singular bodies to Earth $(\sim 3000 \mathrm{~km}$ diameter; Brasser et al. 2016; Day et al. 2016) and Mars ( 1000 km diameter) (Brasser \& Mojzsis 2017; Bouvier et al. 2018) also conveniently explains the coterminous ages of the silicate crusts of these worlds by about 4480 Ma (e.g., Zhang 2002), but evidently not earlier. The low probability of two colossal impacts occurring nearly simultaneously on both Earth and Mars (Brasser et al. 2016) provides circumstantial evidence in support of giant planet migration as a trigger. Finally, giant planet migration must have happened within the first $100 \mathrm{Myr}$ 
of solar system history to account for the survival of Jupiter Trojan binary asteroids (Nesvorný et al. 2018).

\subsection{Life Could Have Emerged on Earth Any Time after $4400 \mathrm{Ma}$}

Our reconciled timeline for late accretion to the inner solar system now leads us to explore arguments for and against the existence of a persistent biosphere on the early Hadean Earth (Mojzsis et al. 1996; Ryder 2002).

Building on related works (Maher \& Stevenson 1988; Melosh 1989; Abramov \& Mojzsis 2009; Abramov et al. 2013; Marchi et al. 2014), we show that annihilation of the terrestrial surface zone (including wholesale melting of the lithosphere) before $\sim 4450$ Ma was such that no stable niches for the prebiotic chemistry leading to life were possible in the first $\sim 100 \mathrm{Myr}$. If the last global-scale crustal melting on Earth corresponds to a colossal impact at ca. 4450 (minimum mass required is $9.31 \times$ $10^{21} \mathrm{~kg}$ ), such an event effectively sterilized the planet by eroding the hydrosphere, melting the crust and creating shallow magma oceans (e.g., Schlichting \& Mukhopadhyay 2018). To further elaborate on how late accretion affects the nascent surface biosphere, we use a new suite of global 3D analytical codes to analyze the global thermal fields of impacts in the time frame 4500-3500 Ma.

Results show that an abating impact flux from late accretion is inadequate to sterilize the surface zone-defined here as $\geqslant 130^{\circ} \mathrm{C}$ for the volume of the upper $4 \mathrm{~km}$ of the crust-after about $4400 \mathrm{Ma}$. This estimate leads to a quantitative approximation for the time of life's origin on the Hadean Earth. If the shift from the nonliving to living world included an RNA biome as a transitional form inhabiting relatively mild aqueous near- or at-surface regions (Mojzsis et al. 2001; Powner et al. 2009; Benner et al. 2012; Becker et al. 2018; see Martin et al. 2008), such fragile proto-organisms (Bernhardt 2012) were susceptible to extinction from successive regional thermal stresses to the hydrosphere from the largest impacts. In light of this, we speculate that the successors to the RNA World-those thermally more robust DNA-peptide microbial immigrants adapted to colonize deep and hot crustal settings near hydrothermal vents and well away from a surface zone bombarded by impacts and bathed in intense ultraviolet radiation from the young Sun-endured the waning stages of bombardment to repopulate the planet from safe havens within the crust.

\section{Summary}

The giant planets radially migrated after dissipation of the protoplanetary nebula around the young Sun, yet the timing of this migration is unconstrained. Here we show that radiometric ages from various meteorite classes can be reconciled with giant planet migration if it occurred before $4480 \mathrm{Ma}$. Our result resolves crater chronologies with late accretion timescales and dynamic models. It also implies that conditions on the Hadean Earth during late accretion do not preclude the emergence of an enduring biosphere as early as $\sim 170$ million years after solar system formation.

This work was funded by the Collaborative for Research in Origins (CRiO), which is supported by The John Templeton Foundation (principal investigator: Steven Benner/FfAME); the opinions expressed in this publication are those of the authors and do not necessarily reflect the views of the John Templeton Foundation. S.J.M. and N.M.K. were supported by the NASA Cosmochemistry program (grant No. NNX14AG31G) in part of this work. S.J.M. thanks the Earth-Life Science Institute (ELSI) at the Tokyo Institute of Technology and the Geophysical Fluid Dynamics Group at the Swiss Federal Institute of Technology (ETH) in Zürich, on visiting professorial appointments during significant phases of this research. R.B. is grateful for financial support from JSPS Kakenhi (17KK0089). O.A. and S.J.M. thank the NASA Solar System Workings Program, grant No. 80NSSC17K0732. S.C.W. appreciates the support by the Research Council of Norway via the Centre of Excellence grant to the Centre of Earth Evolution and Dynamics (CEED 223272). We thank Steven Benner, Thomas Carell, Guillaume Caro, Nicolas Dauphas, Vera Fernandes, Bernard Marty, Michael Russell, and Grenville Turner for discussions. Editorial handling by Michael Endl and reviews by an anonymous reviewer helped to improve this work.

\section{Author Contributions}

S.J.M. formulated the study, supervised all work, interpreted data, and wrote the manuscript. R.B. developed dynamical models of accretion, helped design the study, analyzed and interpreted the results from the dynamical models, and cowrote the manuscript. N.M.K. compiled the thermochronologies of solar system objects. O.A. wrote the revised analytical thermal bombardment codes and ran simulations of the crustal evolution of the Hadean Earth using our new impact bombardment production functions. S.C.W. performed supportive crater statistical analyses and shared her crater chronology data.

The authors declare no conflict of interest.

\section{ORCID iDs}

Stephen J. Mojzsis i https://orcid.org/0000-0003-0000-125X

\section{References}

Abramov, O., Kring, D. A., \& Mojzsis, S. J. 2013, ChEG, 73, 227

Abramov, O., \& Mojzsis, S. J. 2009, Natur, 459, 419

Albarède, F., \& Juteau, M. 1984, GeCoA, 48, 207

Allègre, C. J., Manhès, G., \& Göpel, C. 1995, GeCoA, 59, 1445

Allègre, C. J., Manhès, G., \& Göpel, C. 2008, E\&PSL, 267, 386

Barboni, M., Boehnke, P., Keller, B., et al. 2017, SciA, 3, e1602365

Becker, S., Schneider, C., Okamura, H., et al. 2018, NatCo, 9, 163

Bekaert, D. V., Avice, G., Marty, B., Henderson, B., \& Gudipati, M. S. 2017, GeCoA, 218, 114

Benner, S. A., Kim, H. J., \& Yang, Z. 2012, Cold Spring Harbor Perspectives in Biology, 4, a003541

Bernhardt, H. S. 2012, Biology Direct, 7, 23

Boehnke, P., \& Harrison, T. M. 2016, PNAS, 113, 10802

Bogard, D. D. 1995, M\&PS, 30, 244

Bollard, J., Connelly, J. N., Whitehouse, M. J., et al. 2017, SciA, 3, e1700407

Borg, L. E., Connelly, J. N., Boyet, M., \& Carlson, R. W. 2011, Natur, 477, 70

Borg, L. E., Gaffney, A. M., \& Shearer, C. K. 2015, M\&PS, 50, 715

Bottke, W. F., Durda, D. D., Nesvorný, D., et al. 2005, Icar, 179, 63

Bottke, W. F., Vokrouhlicky, D., Minton, D., et al. 2012, Natur, 485, 78

Bottke, W. F., Walker, R. J., Day, J. M. D., Nesvorný, D., \& Elkins-Tanton, L. 2010, Sci, 330, 1527

Bouvier, L. C., Costa, M. M., Connelly, J. N., et al. 2018, Natur, 558, 568

Boyet, M., \& Carlson, R. W. 2007, E\&PSL, 262, 505

Brasser, R., Dauphas, N., \& Mojzsis, S. J. 2018, GeoRL, 45, 5908

Brasser, R., \& Lee, M. H. 2015, AJ, 150, 157

Brasser, R., \& Mojzsis, S. J. 2017, GeoRL, 44, 5978

Brasser, R., Mojzsis, S. J., Matsumura, S., \& Ida, S. 2017, E\&PSL, 468, 85 
Brasser, R., Mojzsis, S. J., Werner, S. C., Matsumura, S., \& Ida, S. 2016, E\&PSL, 455, 85

Brasser, R., \& Morbidelli, A. 2013, Icar, 225, 40

Brasser, R., Morbidelli, A., Gomes, R., Tsiganis, K., \& Levison, H. F. 2009, A\&A, 507, 1053

Chyba, C. F. 1990, Natur, 343, 129

Clement, M.-S., Kaib, N.-A., \& Raymond, S.-N. 2019, Icar, 321, 778

Connelly, J. N., \& Bizzarro, M. 2016, E\&PSL, 452, 36

Day, J. M. D., Brandon, A. D., \& Walker, R. J. 2016, RvMG, 81, 161

DeMeo, F. E., \& Carry, B. 2014, Natur, 505, 629

Di Sisto, R. P., Fernández, J. A., \& Brunini, A. 2009, Icar, 203, 140

Dodson, M. H. 1973, CoMP, 40, 259

Duncan, M. J., Levison, H. F., \& Lee, M. H. 1998, AJ, 116, 2067

Fernandes, V. A., Fritz, J., Weiss, B. P., Garrick-Bethell, I., \& Shuster, D. L. 2013, M\&PS, 48, 241

Fernandez, J. A., \& Ip, W.-H. 1984, Icar, 58, 109

Fraser, W. C., \& Kavelaars, J. J. 2009, AJ, 137, 72

Fritz, J., Bitsch, B., Kührt, E., et al. 2014, P\&SS, 98, 254

Genda, H., \& Abe, Y. 2005, Natur, 433, 842

Genda, H., Brasser, R., \& Mojzsis, S. J. 2017, E\&PSL, 480, 25

Gomes, R., Levison, H. F., Tsiganis, K., \& Morbidelli, A. 2005, Natur, 435,466

Greenwood, J. P., Itoh, S., Sakamoto, N., et al. 2011, NatGe, 4, 79

Greenwood, J. P., Karato, S., Van der Kaaden, K. E., Pahlevan, K., \& Usui, T. 2018, SSRv, 214, 92

Hansen, B. M. S. 2009, ApJ, 703, 1131

Hartmann, W. K. 1970, Icar, 12, 131

Hartmann, W. K. 2003, M\&PS, 38, 579

Hartmann, W. K., Ryder, G., Dones, L., \& Grinspoon, D. H. 2000, in Origin of the Earth and Moon, ed. R. M. Canup \& K. Righter (Tucson, AZ: Univ. Arizona Press), 493

Heisinger, H., \& Head, J. W. 2006, RvMG, 60, 1

Hopkins, M., Harrison, T. M., \& Manning, C. E. 2008, Natur, 456, 493

Hopkins, M., Harrison, T. M., \& Manning, C. E. 2010, E\&PSL, 298, 367

Hopkins, M. D., \& Mojzsis, S. J. 2015, CoMP, 169, 30

Hopkins, M. D., Mojzsis, S. J., Bottke, W. F., \& Abramov, O. 2015, Icar, 245,367

Humayun, M., Nemchin, A., Zanda, B., et al. 2013, Natur, 503, 513

Ireland, T. R., \& Wlotzka, F. 1992, E\&PSL, 109, 1

Izidoro, A., Haghighipour, N., Winter, O. C., \& Tsuchida, M. 2014, ApJ, 782,31

Johnson, B. C., Collins, G. S., Minton, D. A., et al. 2016, Icar, 271, 350

Kelly, N. M., Metcalf, J. R., Flowers, R. M., \& Mojzsis, S. J. 2018, E\&PSL, 482,222

Kieffer, S. W., \& Simonds, C. H. 1980, RvGSP, 18, 143

Kring, D. A., \& Cohen, B. A. 2002, JGRE, 107, E2

Levison, H. F., \& Duncan, M. J. 1994, Icar, 108, 18

Levison, H. F., \& Duncan, M. J. 1997, Icar, 127, 13

Levison, H. F., Morbidelli, A., Tsiganis, K., Nesvorný, D., \& Gomes, R. 2011, AJ, 142,152

Lichtenberg, T., Golabek, G. J., Gerya, T. V., \& Meyer, M. R. 2016, Icar, 274, 350

Lock, S. J., Stewart, S. T., Petaev, M. L., et al. 2018, JGRE, 123, 910

Maher, K. A., \& Stevenson, D. J. 1988, Natur, 331, 612

Malhotra, R. 1993, Natur, 365, 819

Malhotra, R. 1995, AJ, 110, 420

Marchi, S., Bottke, W. F., Elkins-Tanton, L. T., et al. 2014, Natur, 511, 578

Marchi, S., Bottke, W. F., Kring, D. A., \& Morbidelli, A. 2012, E\&PSL, 325,27

Marchi, S., Chapman, C. R., Fassett, C. I., et al. 2013, Natur, 499, 59

Martin, W., Baross, J., Kelley, D., \& Russell, M. J. 2008, Nature Reviews Microbiology, 6, 805

Marty, B., Altwegg, K., Balsiger, H., et al. 2017, Sci, 356, 1069

McKinnon, W. B., Zahnle, K. J., Ivanov, B. A., \& Melosh, H. J. 1997, in In Venus II Geology, Geophysics, Atmosphere and Solar Wind Environment, ed. S. W. Bougher, D. M. Hunten, \& R. J. Phillips (Tucson, AZ: Univ. Arizona Press), 585

Melosh, H. J. 1989, Impact Cratering. A Geologic Process (Oxford: Clarendon Press), 245

Mojzsis, S. J., Arrhenius, G., McKeegan, K. D., et al. 1996, Natur, 384, 55

Mojzsis, S. J., Harrison, T. M., \& Pidgeon, R. T. 2001, Natur, 409, 178

Morbidelli, A., Brasser, R., Gomes, R., Levison, H. F., \& Tsiganis, K. 2010, AJ, 140, 1391

Morbidelli, A., Marchi, S., Bottke, W. F., \& Kring, D. A. 2012, E\&PSL, 355,144

Morbidelli, A., Nesvorný, D., Laurenz, V., et al. 2018, Icar, 305, 262

Morris, M. A., Garvie, L. A. J., \& Knauth, L. P. 2015, ApJL, 801, L22

Nemchin, A. A., Timms, N., Pidgeon, R., et al. 2009, NatGe, 2, 133

Nesvorný, D. 2011, ApJL, 742, L22

Nesvorný, D. 2015a, AJ, 150, 68

Nesvorný, D. 2015b, AJ, 150, 73

Nesvorný, D. 2018, ARA\&A, 56, 137

Nesvorný, D., \& Morbidelli, A. 2012, AJ, 144, A117

Nesvorný, D., Vokrouhlicky, D., Bottke, W. F., \& Levison, H. F. 2018, NatAs, 2, 878

Neukum, G., Ivanov, B. A., \& Hartmann, W. K. 2001, SSRv, 96, 55

Neukum, G., Koenig, B., \& Arkani-Hamed, J. 1975, Moon, 12, 201

Norman, M. D., \& Nemchin, A. A. 2014, E\&PSL, 388, 387

Öpik, E. J. 1960, MNRAS, 120, 404

Peale, S. J. 1989, Icar, 82, 36

Petro, N. E., \& Pieters, C. M. 2006, JGRE, 111, 9005

Pierazzo, E., \& Melosh, H. J. 2000, Icar, 145, 252

Powner, M. W., Gerland, B., \& Sutherland, J. D. 2009, Natur, 459, 239

Reiners, P. W., Ehlers, T. A., \& Zeitler, P. K. 2005, RvMG, 58, 1

Reiners, P. W., Shuster, D. L., \& Evenson, N. 2015, AGU Fall Meeting, V53E-3165

Rolf, T., Steinberger, B., Sruthi, U., \& Werner, S. C. 2018, Icar, 313, 107

Roth, A. S. G., Bourdon, B., Mojzsis, S. J., et al. 2014, GGG, 15, 2329

Ryder, G. 1990, Eos, 71, 313

Ryder, G. 2002, JGR, 107, 5022

Schlichting, H. E., \& Mukhopadhyay, S. 2018, SSRv, 214, 34

Shearer, C., \& Borg, L. 2006, ChEG, 66, 163

Shoemaker, E. M., Hackman, R. J., \& Eggleton, R. E. 1962, AdAnS, 8, 70

Strom, R. G., Malhotra, R., Ito, T., Yoshida, F., \& Kring, D. A. 2005, Sci, 309, 1847

Strom, R. G., \& Neukum, G. 1988, in The Cratering Record on Mercury and the Origin of Impacting Objects, ed. F. Vilas, C. Chapman, \& M. Matthews (Tucson, AZ: Univ. Arizona Press), 336

Tera, F., Papanastassiou, D. A., \& Wasserburg, G. J. 1974, E\&PSL, 22, 1

Thommes, E. W., Duncan, M. J., \& Levison, H. F. 1999, Natur, 402, 635

Tremaine, S., \& Dones, L. 1993, Icar, 106, 335

Tsiganis, K., Gomes, R., Morbidelli, A., \& Levison, H. F. 2005, Natur, 435,459

Turner, G., Cadogan, P. H., \& Yonge, C. J. 1973, Natur, 242, 513

Valley, J. W., Cavosie, A. J., Ushikubo, T., et al. 2014, NatGe, 7, 219

Walker, R. J. 2009, ChEG, 69, 101

Walsh, K. J., Morbidelli, A., Raymond, S. N., O’Brien, D. P., \& Mandell, A. M. 2011, Natur, 475, 206

Werner, S. C. 2014, E\&PSL, 400, 54

Werner, S. C., \& Ivanov, B. A. 2015, Treatise on Geophysics, Vol. 10 (2nd ed; Amsterdam: Elsevier), 327

Wetherill, G. W. 1967, JGR, 72, 2429

Wielicki, M. M., Harrison, T. M., \& Schmitt, A. K. 2012, E\&PSL, 321, 20

Wong, E. W., Brasser, R., \& Werner, S. C. 2019, E\&PSL, 506, 407

Woo, J. M. Y., Brasser, R., Matsumura, S., Mojzsis, S. J., \& Ida, S. 2018, A\&A, 617, 17

Woo, J. M. Y., Genda, H., Brasser, R., \& Mojzsis, S. J. 2019, Icar, 333, 87

Zellner, N. E. B. 2017, OLEB, 47, 261

Zhang, Y. 2002, ESRv, 59, 235

Zhou, Q., Yin, Q.-Z., Young, E. D., et al. 2013, GeCoA, 110, 152 